\title{
Multi-platform characterization of the human cerebrospinal fluid metabolome: a comprehensive and quantitative update
}

\author{
Rupasri Mandal', An Chi Guo², Kruti K Chaudhary', Philip Liu', Faizath S Yallou', Edison Dong ${ }^{1}$, Farid Aziat ${ }^{1}$ and \\ David S Wishart ${ }^{1,2,3^{*}}$
}

\begin{abstract}
Background: Human cerebral spinal fluid (CSF) is known to be a rich source of small molecule biomarkers for neurological and neurodegenerative diseases. In 2007, we conducted a comprehensive metabolomic study and performed a detailed literature review on metabolites that could be detected (via metabolomics or other techniques) in CSF. A total of 308 detectable metabolites were identified, of which only $23 \%$ were shown to be routinely identifiable or quantifiable with the metabolomics technologies available at that time. The continuing advancement in analytical technologies along with the growing interest in CSF metabolomics has led us to re-visit the human CSF metabolome and to re-assess both its size and the level of coverage than can be achieved with today's technologies.

Methods: We used five analytical platforms, including nuclear magnetic resonance (NMR), gas chromatographymass spectrometry (GC-MS), liquid chromatography-mass spectrometry (LC-MS), direct flow injection-mass spectrometry (DFI-MS/MS) and inductively coupled plasma - mass spectrometry (ICP-MS) to perform quantitative metabolomics on multiple human CSF samples. This experimental work was complemented with an extensive literature review to acquire additional information on reported CSF compounds, their concentrations and their disease associations.
\end{abstract}

Results: NMR, GC-MS and LC-MS methods allowed the identification and quantification of 70 CSF metabolites (as previously reported). DFI-MS/MS allowed the quantification of 78 metabolites (6 acylcarnitines, 13 amino acids, hexose, 42 phosphatidylcholines, 2 lyso-phosphatidylcholines and 14 sphingolipids), while ICP-MS provided quantitative results for 33 metal ions in CSF. Literature analysis led to the identification of 57 more metabolites. In total, 476 compounds have now been confirmed to exist in human CSF.

Conclusions: The use of improved metabolomic and other analytical techniques has led to a 54\% increase in the known size of the human CSF metabolome over the past 5 years. Commonly available metabolomic methods, when combined, can now routinely identify and quantify 36\% of the 'detectable' human CSF metabolome. Our experimental works measured 78 new metabolites that, as per our knowledge, have not been reported to be present in human CSF. An updated CSF metabolome database containing the complete set of 476 human CSF compounds, their concentrations, related literature references and links to their known disease associations is freely available at the CSF metabolome database.

\footnotetext{
* Correspondence: dwishart@ualberta.ca

${ }^{1}$ Department of Biological Sciences, University of Alberta, 11455

Saskatchewan Drive, Edmonton, AB Canada T6G 2E8

Full list of author information is available at the end of the article
} 


\section{Background}

There is a growing need among the metabolomics and clinical communities to develop comprehensive, centralized reference resources for clinically important biofluids such as cerebrospinal fluid, blood, urine and saliva. In this regard, we have undertaken the task to systematically characterize each of these biofluids as part of the human metabolome project [1]. The first biofluid we studied in detail, in 2007, was human cerebrospinal fluid (CSF) [2]. Although CSF is not an easily accessible biofluid, its relative metabolic simplicity and potential importance to central nervous system diseases makes it particularly important in biomedical research and clinical chemistry [2]. Since the composition of CSF is directly dependent upon metabolite production rates in the brain [3], analysis of the CSF metabolome can potentially offer biochemical insights into central nervous system disorders, such as brain injury [4], Alzheimer's disease [5], Parkinson's disease [6] and multiple sclerosis [7]. Indeed, in the five years since our initial 'CSF metabolome' study was completed the CSF metabolome database [8] has been used to facilitate a wide range of metabolomic studies on central nervous system diseases, including multiple sclerosis [9], brain cancer [10] and amyotrophic lateral sclerosis [11].

At the time it was first published, the CSF metabolome database consisted of a total of 308 detectable metabolites, with extensive information on compound names, structures, identifiers, concentrations, related literature references and links to known disease associations. In that CSF study [2], we also showed that the metabolomic technologies available at that time were able to detect and quantify only about $23 \%$ of the known or detectable CSF compounds. Since that time, continuing advances in the analytical technologies for metabolomics (including improvements to instrumentation sensitivity, enhanced separation capacity, better software and more compound standards) have occurred. This technical improvement, along with the growing interest in the CSF metabolome in clinical communities has led us to re-visit the human CSF metabolome. In particular we wanted to find out if these improved technologies could lead to a substantive improvement to the level of CSF metabolite coverage achievable by standard metabolomic technologies. We also wanted to determine if new, or previously unidentified, CSF metabolites had been reported in the literature or could be discovered using these enhanced metabolomics platforms. Finally, we wanted to update the CSF metabolome database so that it contained the latest information on all known or detectable CSF metabolites, their concentrations, the latest references and their disease associations.

Here we wish to report the results of this work, including the use of five different metabolomic platforms (nuclear magnetic resonance (NMR), gas chromatography-mass spectrometry (GC-MS), liquid chromatography-mass spectrometry (LC-MS), direct flow injection-mass spectrometry (DFI-MS/MS) and inductively coupled plasma-mass spectrometry (ICP-

MS)) to characterize multiple CSF samples as well as an extensive literature review (covering the period from 2008 to 2011) aimed at identifying and tabulating new (or previously unidentified) CSF metabolites along with new or updated CSF metabolite biomarkers. All of these data, along with their concentrations, related literature references and links to their known disease associations are freely available at the CSF metabolome database [8].

\section{Materials and methods}

\section{Cerebral spinal fluid samples}

Lumbar CSF samples were collected from patients screened for meningitis in accordance with guidelines and consent protocols established by the University of Alberta Research Ethics Board [2] and conforming to the Declaration of Helsinki principles. Only a small portion $(<5 \%)$ were shown to have meningitis, suggesting that the CSF samples were from mostly neurologically normal individuals.

A more detailed list describing the patient population is shown in Table 1. The typical volume of each CSF samples was 0.5 to $1.0 \mathrm{ml}$. CSF samples were placed in a freezer for long-term storage at $-80^{\circ} \mathrm{C}$. All CSF samples were thawed on ice for approximately $2 \mathrm{~h}$ before use. A total of seven samples were used for the analyses described below.

\section{NMR, GC-MS and LC-MS compound identification and quantification}

Identical procedures using identical instruments, as described in [2], were employed to process and characterize metabolites from the CSF samples collected above. More specifically, all ${ }^{1} \mathrm{H}-\mathrm{NMR}$ spectra were collected on $500 \mu \mathrm{l} \mathrm{CSF}$ samples at $25^{\circ} \mathrm{C}$ (via the first transient of the tnnoesy-presaturation pulse sequence) using a $500 \mathrm{MHz}$ Inova (Varian Inc., Palo Alto, CA, USA) spectrometer equipped with a $5 \mathrm{~mm}$ Z-gradient PFG Varian cold-probe. All ${ }^{1} \mathrm{H}-\mathrm{NMR}$ spectra were baseline corrected and analyzed using the Chenomx NMR Suite Professional software package version 6.1 (Chenomx Inc., Edmonton, $A B$, Canada). All GC-MS data were acquired on an HP 6890/

\section{Table 1 Summary of samples}

\begin{tabular}{ll}
$\begin{array}{l}\text { Number of } \\
\text { patients }\end{array}$ & $\mathbf{7}$ \\
\hline Age & Adult (18 to 40 years old) \\
Race & White \\
Disease & $\begin{array}{l}\text { Screened for meningitis; only a small portion }(<5 \%) \\
\text { were shown to have meningitis }\end{array}$ \\
\hline
\end{tabular}


5975 GC/MS equipped with a DB-5 column. N-methyl-N(trimethylsilyl) trifluoroacetamide (MSTFA) derivatized extracts were prepared from $200 \mu \mathrm{l}$ of CSF using standard methods [2]. Samples were run using full scan at a mass range of 50 to $500 \mathrm{~m} / \mathrm{z}$, with a 55 minute run time using a starting temperature of $70^{\circ} \mathrm{C}$ and a final temperature of $350^{\circ} \mathrm{C}$. Trimethylsilated metabolites were identified using the AMDIS (automated mass spectral deconvolution and identification system) software package [12] in conjunction with the 2008 National Institute of Standards and Technology (NIST) library [13], and quantified using external multi-point calibration curves. For the LC-MS studies, CSF samples were pooled and analyzed using a Bruker Daltonics 9.4T Apex-Qe FT-ICR mass spectrometer equipped with a Waters ultra-performance liquid chromatography (UPLC) system. Spectra were collected in both positive and negative ion modes. Metabolites were identified and confirmed by high resolution Fourier transform mass spectrometry (FTMS by comparing their parent ion and fragment ion masses to known masses or fragment ion spectra from the Human Metabolome Database [14].

\section{DFI-MS/MS compound identification and quantification}

In addition to characterizing CSF using NMR, GC-MS and LC-FTMS, we also employed a targeted quantitative metabolomics approach using direct flow injection mass spectrometry (AbsoluteIDQ ${ }^{\mathrm{TM}} \mathrm{kit}$ ). The kit is a commercially available assay from Biocrates Life Sciences AG (Innsbruck, Austria), and it was originally validated for plasma samples. Recently, the kit has been optimized for the analysis of human CSF [15] and urine samples. This kit assay, in combination with a 4000 QTrap (Applied Biosystems/MDS Sciex, Concord, Ontario, Canada) mass spectrometer, was used to identify and quantify of a large number of endogenous metabolites, including amino acids, acylcarnitines, glycerophospholipids, sphingolipids and sugars. The method combines the derivatization and extraction of analytes with the selective mass-spectrometric detection using multiple reaction monitoring (MRM) pairs. Isotope-labeled internal standards are integrated into a kit plate filter for metabolite quantification.

The AbsoluteIDQTM kit contains a 96 deep-well plate with a filter plate attached with sealing tape, as well as reagents and solvents used to prepare the plate assay. The first eight wells in each kit are used for standardization and quality control. A straightforward sample preparation step was used for the assay, as described in the kit's user manual. CSF samples were left to thaw on ice, and then vortexed and centrifuged at 13,000 $\times \mathrm{g}$. A total of $30 \mu \mathrm{l}$ of supernatant from each CSF sample was loaded on a filter paper placed on top of the kit plate and dried in a stream of nitrogen. Subsequently, $20 \mu \mathrm{l}$ of a $5 \%$ solution of phenyl-isothiocyanate was added for derivatization. After incubation, the filter spots were dried again using an evaporator. Extraction of the metabolites was then achieved by adding $300 \mu \mathrm{l}$ methanol containing $5 \mathrm{mM}$ ammonium acetate. The extracts were obtained by centrifugation into the lower 96-deep well plate, followed by a dilution step with $600 \mu \mathrm{l}$ of the kit's mass spectrometry running solvent. The extracts were analyzed using a 4000 QTrap (Applied Biosystems/MDS Sciex) mass spectrometer. A standard flow injection protocol consisting of two $20 \mu$ injections (one for the positive and one for the negative ion detection mode) was applied for all measurements. MRM detection was used for quantification. MetIQ software, which is proprietary to Biocrates and included in the kit, was used to control the entire assay workflow. This included sample registration to automated calculation of metabolite concentrations to the export of data into other data analysis programs.

\section{Multi-element analysis using ICP-MS}

For elemental (primarily metal) analysis by ICP-MS, seven CSF samples were processed as described previously [16]. In particular, CSF samples were sonicated in an ultrasound water bath for 10 minutes in order to obtain a homogeneous dispersion. The sample was then diluted with $2 \%$ $\mathrm{HNO}_{3}$. Elemental concentrations were determined on a Perkin-Elmer Sciex Elan 6000 quadrupole ICP-MS operating in a dual detector mode (Santa Clara, California, USA). Blank subtraction was applied after internal standard correction. A four point calibration curve was used to quantify compounds $(0,0.025,0.050$, and $0.100 \mathrm{ppm}$ for $\mathrm{Na}$; 0 , 0.25, 0.50, and $1.00 \mathrm{ppm}$ for $\mathrm{Ca}, \mathrm{Mg}, \mathrm{Fe}, \mathrm{K} ; 0,0.005,0.010$, and $0.020 \mathrm{ppm}$ for the remaining elements). The sample uptake rate was approximately $1 \mathrm{ml} /$ minute with 35 sweeps per reading using one reading per replicate and three replicates. Dwell times were 10 to $20 \mathrm{~ms}$ for all elements with the exception of As (which was $100 \mathrm{~ms}$ ). The relative standard deviation ( $2 \sigma$ level) for $\mathrm{As}, \mathrm{Ni}, \mathrm{Pb}$, and $\mathrm{Zn}$ was between 5 and $10 \%$. The accuracy of the ICP-MS analytical protocol was periodically evaluated via the analysis of certified reference standard materials (whole rock powders) BE-N and DR-N available from the SARM laboratory at the CRPG (Centre de Recherches Pétrographiques et Géologiques).

\section{Literature survey of CSF metabolites}

In addition to the experimental analysis of the CSF metabolome described above, a comprehensive literature review, covering the past 4 years (2008 to 2011) was performed to look at known or newly discovered CSF metabolites and metabolite concentrations. An in-house text-mining tool, which was originally developed for the Human Metabolome Database [14], was used to facilitate the literature survey. This program generated a hyperlinked list of abstracts and papers from PubMed containing relevant information 
about CSF metabolites and CSF concentration data. Keywords used for this literature search included terms such as 'CSF', 'cerebrospinal fluid', 'human', 'concentration', 'quantification' and the names of the metabolites in the Human Metabolome Database. From the resulting 106 papers and abstracts, we manually extracted metabolite information (metabolite identities, concentrations, associated disease states, and so on) and entered the data into our CSF metabolome database.

\section{Results and discussion}

NMR, GC-MS and LC-MS compound identification and quantification

A total of 53 metabolites were identified (47 quantified) by NMR, 41 metabolites were identified (15 quantified) by GC-MS, and LC-MS permitted the identification of 17 metabolites. A total of 70 non-redundant CSF metabolites were identified (62 quantified) using these three platforms.
No additional metabolites beyond those originally reported in [2] were identified. No doubt the use of more modern equipment might have improved the situation, but certainly when considering NMR as a metabolomic platform, CSF spectra appear to be fully determined and fully assigned. A typical $500 \mathrm{MHz}{ }^{1} \mathrm{H}-\mathrm{NMR}$ spectrum and high resolution GC-MS total ion chromatogram are shown in Figures 1 and 2, respectively.

\section{DFI-MS/MS compound identification and quantification}

The Biocrates AbsoluteIDQTM kit permits the measurement of 162 metabolites (41 acylcarnitines, 14 amino acids, hexose, 76 phospatidylcholines (PCs), 15 lyso-phosphatidylcholines and 15 sphingolipids). From these 162 potentially detectable metabolites, quantitative results were obtained for 78 metabolites ( 6 acylcarnitines, 13 amino acids, hexose, 42 PCs, 2 lyso-phosphatidylcholines and 14 sphingolipids). These results are summarized in

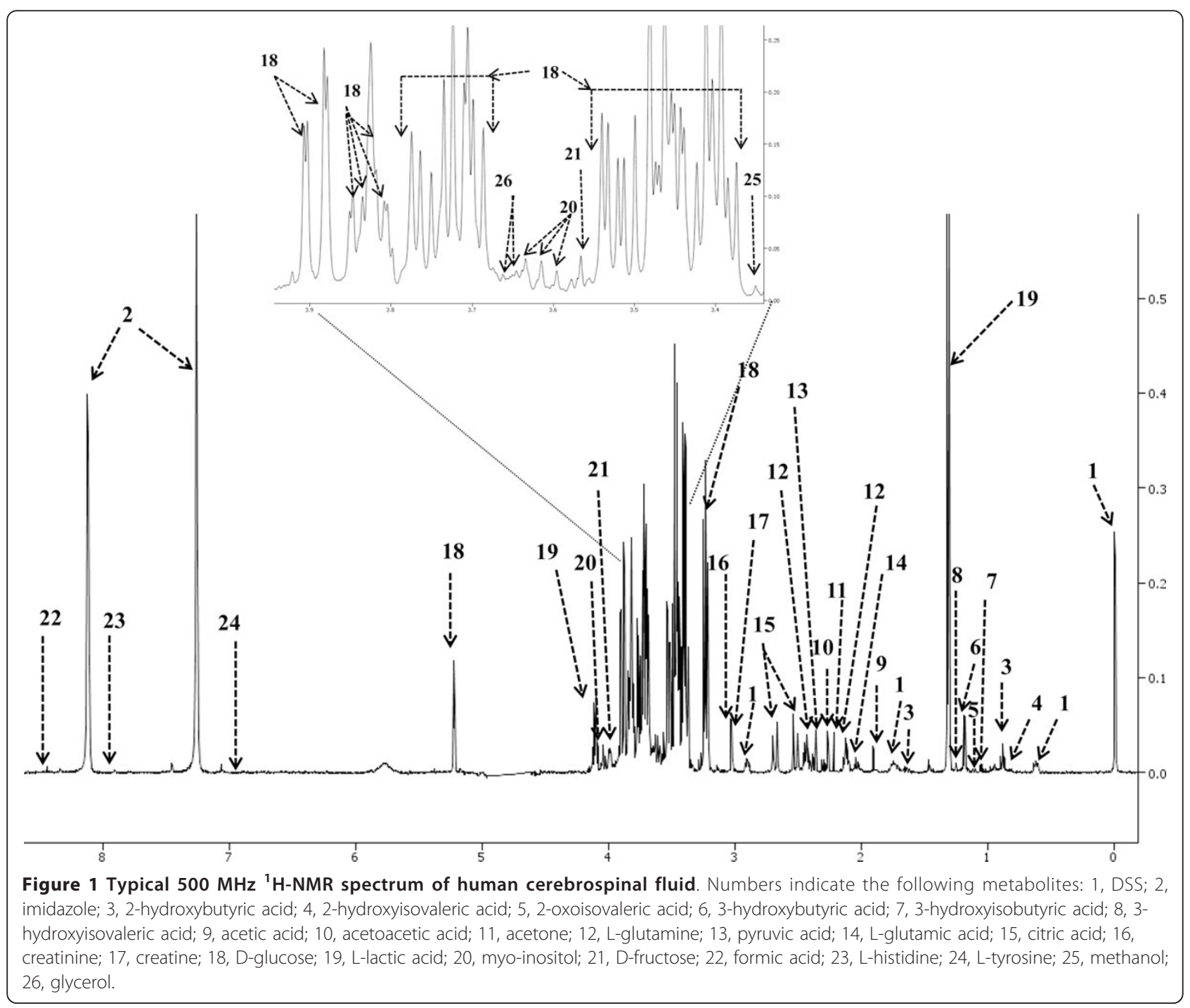




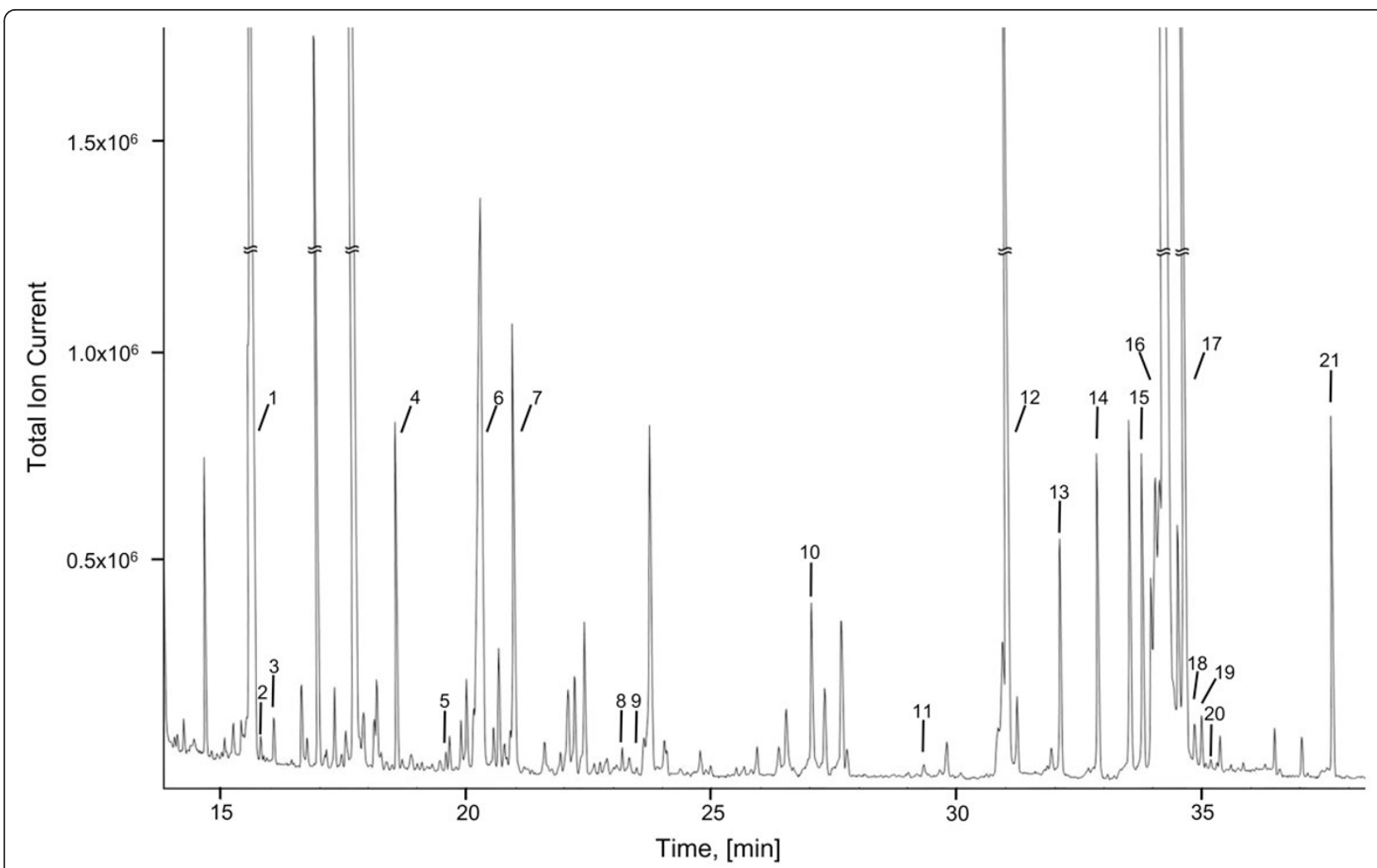

Figure 2 Typical GC-MS total ion chromatogram spectrum of human cerebrospinal fluid. Numbers indicate the following metabolites: 1, Llactic acid; 2, oxalic acid; 3, L-alanine; 4, glycine; 5, L-valine; 6, urea; 7, glycerol; 8, L-serine; 9, L-threonine/pyroglutamic acid; 10, L-glutamine; 11, Lphenylalanine; 12, ribitol; 13, L-glutamic acid; 14, citric acid; 15, D-fructose; 16, D-glucose; 17, D-galactose; 18, L-lysine; 19, mannitol; 20, L-tyrosine; 21, myo-inositol.

Table 2 and typical direct flow injection (DFI) spectra in both positive and negative modes are shown in Figure 3. The other 84 metabolites that were assayed were below the limit of detection. This result is in agreement with a previous study conducted by Biocrates (Application Note 1003-1 [15]) for pooled human CSF samples that reported a total of 65 quantified metabolites (5 acylcarnitines, 14 amino acids, hexose, 35 PCs and 10 sphingolipids).

Of the 78 metabolites quantified by DFI-MS/MS, 11 compounds (10 amino acids and hexose/glucose) were also measurable using NMR and/or GC-MS. The measured concentrations for these common compounds on all three platforms showed very good agreement (within 15 to $30 \%$ of each other). More importantly, the DFI-MS/MS method provided quantitative data on 67 unique compounds that GC-MS, LC-MS and NMR methods could not detect. It is important to note, however, that DFI-MS/ MS generates phospholipid data (PCs) that only identifies PCs by their total acyl/alkyl chain content (for example, $\mathrm{PC}$ aa 38:4) rather than their precise chemical structure. These $42 \mathrm{PC}$ 'species' along with their concentrations are entered in our CSF metabolome database. In addition, each PC species is linked to a list of the most probable PC structures (586 in total) based on the known fatty acid compositions in humans.

\section{Multi-element analysis using ICP-MS}

Trace metals are known to play an important role in enzyme function and a wide number of neurodegenerative diseases like Alzheimer's disease, Parkinson's disease and other related diseases [17]. A new trend in metabolomics (called 'metallomics') is evolving wherein trace metal concentrations of tissues, biofluids and even individual cells are measured. It is essential to measure trace metal concentrations in tissues and body fluids to be used as reference values. However, less data are available for normal or reference values of element concentrations for CSF samples due to the difficulties in accessing the samples and low concentrations of elements. In a recent review the importance of trace metal analysis and the potential of metal speciation analysis in CSF as a diagnostic tool for better understanding of neurodegenerative diseases were discussed [18]. An overview of current analytical techniques (like ICP-MS, inductively coupled plasma optical emission spectrometry, atomic absorption spectroscopy) and the results of total concentrations and 
Table 2 Concentrations of metabolites in human cerebrospinal fluid samples

\begin{tabular}{|c|c|c|c|}
\hline Compound name & Average $(\mu \mathrm{M})$ & Standard deviation $(\mu \mathrm{M})$ & Literature value $(\mu \mathrm{M})$ \\
\hline 1,5-Anhydrosorbitol ${ }^{\mathrm{a}}$ & 25 & 13 & $18 \pm 5$ \\
\hline 2-Hydroxybutyric acid ${ }^{a, b}$ & 40 & 24 & $35 \pm 24$ \\
\hline 2-Hydroxyisovaleric acid ${ }^{\mathrm{a}}$ & 8 & 6 & $7 \pm 7$ \\
\hline 2-Oxoglutaric acid ${ }^{a}$ & 5 & 4 & $9 \pm 3$ \\
\hline 2-Oxoisovaleric acid ${ }^{a}$ & 6 & 3 & $8 \pm 7$ \\
\hline 3-Hydroxybutyric acid ${ }^{a}$ & 34 & 31 & $46 \pm 24$ \\
\hline 3-Hydroxyisobutyric acid ${ }^{a}$ & 6 & 3 & $18 \pm 18$ \\
\hline 3-Hydroxyisovaleric acid ${ }^{a}$ & 4 & 2 & NA \\
\hline Acetaminophen $^{a}$ & 11 & 6 & NA \\
\hline Acetic acid ${ }^{a}$ & 58 & 27 & $100 \pm 30$ \\
\hline Acetoacetic acid ${ }^{a}$ & 12 & 14 & $6 \pm 6$ \\
\hline Acetone $^{a}$ & 20 & 21 & $67 \pm 24$ \\
\hline L-Alanine $^{a}$ & 46 & 27 & $37 \pm 7$ \\
\hline Choline $^{a}$ & 3 & 1 & $8 \pm 5$ \\
\hline Citric acid $^{a}$ & 225 & 96 & $176 \pm 50$ \\
\hline Creatine $^{a}$ & 44 & 13 & NA \\
\hline Creatinine $^{a}$ & 43 & 12 & $65 \pm 25$ \\
\hline Dimethylsulfone $^{a}$ & 2 & 1 & $11 \pm 6$ \\
\hline Dimethylamine $^{a}$ & 2 & 1 & NA \\
\hline Formic acid ${ }^{a}$ & 32 & 16 & NA \\
\hline D-Fructose ${ }^{a}$ & 160 & 91 & $240 \pm 20$ \\
\hline D-Glucose $e^{a_{,} c}$ & 2,960 & 1,110 & $5,390 \pm 1,650$ \\
\hline L-Glutamic acid ${ }^{\mathrm{a}}$ & 40 & 52 & $33 \pm 7$ \\
\hline L-Glutamine $e^{a, c}$ & 398 & 150 & $444 \pm 80$ \\
\hline Glycerol ${ }^{\mathrm{a}, \mathrm{b}}$ & 28 & 14 & $14 \pm 3$ \\
\hline L-Histidine ${ }^{a_{1} c}$ & 15 & 8 & $12 \pm 2$ \\
\hline L-Isoleucine ${ }^{a}$ & 7 & 5 & $8 \pm 3$ \\
\hline Isopropyl alcohol ${ }^{a}$ & 22 & 56 & NA \\
\hline Lactic acid ${ }^{a}$ & 1,651 & 626 & $1,590 \pm 330$ \\
\hline L-Leucine ${ }^{a}$ & 16 & 9 & $19 \pm 4$ \\
\hline L-Lysine ${ }^{a}$ & 29 & 13 & $28 \pm 8$ \\
\hline D-Mannose ${ }^{a}$ & 24 & 13 & $64 \pm 8$ \\
\hline Methanol $^{\mathrm{a}}$ & 44 & 36 & NA \\
\hline L-Methionine $e^{a, c}$ & 5 & 4 & $6 \pm 3$ \\
\hline Myo-inositol $^{a}$ & 84 & 40 & $133 \pm 20$ \\
\hline Oxalacetic acid ${ }^{a}$ & 27 & 15 & $7 \pm 2$ \\
\hline L-Phenyalanine ${ }^{a, c}$ & 15 & 8 & $18 \pm 7$ \\
\hline Propylene glycol $^{a}$ & 33 & 50 & $7.3 \pm 0.5$ \\
\hline Pyroglutamic acid ${ }^{a}$ & 47 & 30 & $41 \pm 30$ \\
\hline Pyruvic acid ${ }^{a}$ & 53 & 42 & $71 \pm 30$ \\
\hline L-Serine $e^{a, c}$ & 38 & 14 & $42 \pm 15$ \\
\hline L-Threonine $e^{a, c}$ & 28 & 8 & $28 \pm 5$ \\
\hline L-Tryptophan a, c & 10 & 5 & $2 \pm 1$ \\
\hline L-Tyrosine $e^{a}$ & 12 & 9 & $10 \pm 4$ \\
\hline L-Valine $^{\mathrm{a}}$ & 19 & 13 & $24 \pm 7$ \\
\hline Xanthine $^{a}$ & 13 & 7 & $5 \pm 1$ \\
\hline 4-Aminobutyric acid ${ }^{b}$ & $<1$ & $<1$ & $0.3 \pm 0.1$ \\
\hline Adenosine $^{\mathrm{b}}$ & $<1$ & $<1$ & $0.01 \pm 0.01$ \\
\hline Ascorbic acid ${ }^{b}$ & 178 & 15 & $164 \pm 20$ \\
\hline L-Asparagine $^{b}$ & 4 & 2 & $5 \pm 1$ \\
\hline L-Aspartic acid ${ }^{\mathrm{b}}$ & 2 & 1 & $3 \pm 1$ \\
\hline
\end{tabular}


Table 2 Concentrations of metabolites in human cerebrospinal fluid samples (Continued)

\begin{tabular}{|c|c|c|c|}
\hline$\overline{D-G_{\text {Galactose }}{ }^{\mathrm{b}}}$ & 107 & 45 & $166 \pm 99$ \\
\hline Mannitol $^{b}$ & 5 & 2 & $5 \pm 1$ \\
\hline Ribitol $^{b}$ & 18 & 5 & $4 \pm 12$ \\
\hline Succinic acid ${ }^{a}$ b & 47 & 12 & $29 \pm 5$ \\
\hline Thymine $^{\mathrm{b}}$ & $<5$ & $<2$ & $<1$ \\
\hline Urea $^{\mathrm{b}}$ & 3,820 & 1,600 & $4,160 \pm 1,800$ \\
\hline Uric acid ${ }^{b}$ & 15 & 10 & $16 \pm 12$ \\
\hline DL-carnitine ${ }^{c}$ & 1.9 & 0.5 & $4.0 \pm 2.0$ \\
\hline Acetyl-L-carnitine ${ }^{c}$ & 0.322 & 0.148 & NA \\
\hline Propionyl-L-carnitine ${ }^{c}$ & 0.018 & 0.009 & NA \\
\hline Hydroxypropionylcarnitine ${ }^{c}$ & 0.063 & 0.030 & NA \\
\hline Butyryl-L-carnitine $^{c}$ & 0.024 & 0.007 & NA \\
\hline Valeryl-L-carnitine ${ }^{c}$ & 0.013 & 0.006 & NA \\
\hline Glycine $^{b, c}$ & 6.1 & 1.4 & $8.2 \pm 3.0$ \\
\hline$L_{\text {-Arginine }}{ }^{c}$ & 17.9 & 5.7 & $20.2 \pm 6.3$ \\
\hline L-Ornithine ${ }^{c}$ & 4.5 & 2.2 & $6.0 \pm 1.5$ \\
\hline L-Proline ${ }^{c}$ & 1.9 & 1.0 & $4.0 \pm 2.0$ \\
\hline $\mathrm{SM}(\mathrm{OH}) \mathrm{C} 14: 1^{\mathrm{C}}$ & 0.028 & 0.004 & NA \\
\hline $\mathrm{SM}(\mathrm{OH}) \mathrm{C} 16: 1^{\mathrm{C}}$ & 0.020 & 0.007 & NA \\
\hline $\mathrm{SM}(\mathrm{OH}) \mathrm{C} 22: 1^{\mathrm{C}}$ & 0.034 & 0.009 & NA \\
\hline $\mathrm{SM}(\mathrm{OH}) \mathrm{C} 22: 2^{\mathrm{c}}$ & 0.032 & 0.008 & NA \\
\hline $\mathrm{SM}(\mathrm{OH}) \mathrm{C} 24: 1^{\mathrm{C}}$ & 0.017 & 0.004 & NA \\
\hline SM C16:0 & 0.336 & 0.109 & NA \\
\hline SM C $16: 1^{c}$ & 0.040 & 0.016 & NA \\
\hline SM C18:0 $0^{c}$ & 0.340 & 0.154 & NA \\
\hline SM C $18: 1^{c}$ & 0.069 & 0.026 & NA \\
\hline SM C20: $2^{c}$ & 0.003 & 0.001 & NA \\
\hline SM C24:0 $0^{C}$ & 0.114 & 0.022 & NA \\
\hline SM C24:1 ${ }^{\mathrm{C}}$ & 0.230 & 0.113 & NA \\
\hline$S M C 26: 0^{c}$ & 0.007 & 0.001 & NA \\
\hline$S M C 26: 1^{c}$ & 0.007 & 0.003 & NA \\
\hline$P C$ aa $C 30: 0^{c}$ & 0.086 & 0.005 & NA \\
\hline PC aa $C 30: 2^{c}$ & 0.010 & 0.004 & NA \\
\hline$P C$ aa $C 32: 0^{c}$ & 0.252 & 0.080 & NA \\
\hline PC aa C32:1 ${ }^{c}$ & 0.150 & 0.051 & NA \\
\hline$P C$ aa $C 32: 2^{c}$ & 0.024 & 0.008 & NA \\
\hline PC aa C34:1 ${ }^{C}$ & 1.800 & 0.490 & NA \\
\hline PC aa $C 34: 2^{c}$ & 0.245 & 0.010 & NA \\
\hline PC aa C34:3 & 0.038 & 0.003 & NA \\
\hline PC aa C36:1 ${ }^{c}$ & 0.212 & 0.062 & NA \\
\hline PC aa $C 36: 2^{c}$ & 0.302 & 0.044 & NA \\
\hline PC aa $C 36: 3^{c}$ & 0.126 & 0.040 & NA \\
\hline$P C$ aa $C 36: 4^{c}$ & 0.228 & 0.036 & NA \\
\hline$P C$ aa $C 36: 5^{c}$ & 0.041 & 0.007 & NA \\
\hline PC aa C38:1 ${ }^{C}$ & 0.041 & 0.002 & NA \\
\hline PC aa $C 38: 3^{c}$ & 0.110 & 0.049 & NA \\
\hline PC aa C38:4 $4^{C}$ & 0.209 & 0.068 & NA \\
\hline$P C$ aa $C 38: 5^{c}$ & 0.074 & 0.011 & NA \\
\hline PC aa C38:6 ${ }^{C}$ & 0.079 & 0.010 & NA \\
\hline PC aa $C 40: 3^{c}$ & 0.004 & 0.001 & NA \\
\hline$P C$ aa $C 40: 4^{c}$ & 0.015 & 0.007 & NA \\
\hline PC aa C40:5 & 0.031 & 0.003 & NA \\
\hline
\end{tabular}


Table 2 Concentrations of metabolites in human cerebrospinal fluid samples (Continued)

\begin{tabular}{|c|c|c|c|}
\hline$\overline{P C}$ aa $C 42: 4^{C}$ & 0.004 & 0.001 & NA \\
\hline$P C$ ae $C 32: 1^{C}$ & 0.022 & 0.007 & NA \\
\hline PC ae C34:0 & 0.018 & 0.005 & NA \\
\hline PC ae C34:1 ${ }^{C}$ & 0.085 & 0.008 & NA \\
\hline PC ae C34:2 & 0.062 & 0.005 & NA \\
\hline$P C$ ae $C 34: 3^{C}$ & 0.011 & 0.001 & NA \\
\hline$P C$ ae $C 36: 1^{C}$ & 0.032 & 0.007 & NA \\
\hline$P C$ ae $C 36: 2^{c}$ & 0.024 & 0.009 & NA \\
\hline$P C$ ae $C 36: 3^{c}$ & 0.017 & 0.006 & NA \\
\hline$P C$ ae $C 36: 4^{c}$ & 0.024 & 0.005 & NA \\
\hline$P C$ ae $C 36: 5^{c}$ & 0.032 & 0.005 & NA \\
\hline$P C$ ae $C 38: 1^{c}$ & 0.009 & 0.002 & NA \\
\hline$P C$ ae $C 38: 2^{c}$ & 0.013 & 0.002 & NA \\
\hline$P C$ ae $C 38: 3^{C}$ & 0.009 & 0.003 & NA \\
\hline$P C$ ae $C 38: 4^{c}$ & 0.020 & 0.005 & NA \\
\hline$P C$ ae $C 38: 5^{c}$ & 0.025 & 0.008 & NA \\
\hline$P C$ ae $C 38: 6^{c}$ & 0.014 & 0.004 & NA \\
\hline$P C$ ae $C 40: 5^{c}$ & 0.006 & 0.001 & NA \\
\hline$P C$ ae $C 40: 6^{c}$ & 0.018 & 0.003 & NA \\
\hline$P C$ ae $C 44: 4^{c}$ & 0.013 & 0.001 & NA \\
\hline PC ae $C 44: 5^{c}$ & 0.024 & 0.009 & NA \\
\hline lysoPC a C18:0 $0^{c}$ & 0.069 & 0.019 & NA \\
\hline lysoPC a C20:4 $4^{c}$ & 0.024 & 0.007 & NA \\
\hline
\end{tabular}

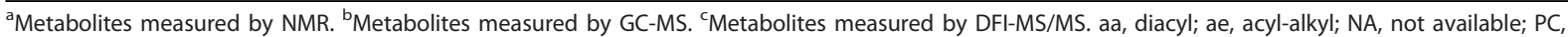
phosphatidylcholine; SM, sphingomyelin.

speciation information for several elements, such as $\mathrm{Al}$, As, $\mathrm{Ca}, \mathrm{Cd}, \mathrm{Cu}, \mathrm{Fe}, \mathrm{Mg}, \mathrm{Mn}, \mathrm{Hg}, \mathrm{Pb}$, Se and $\mathrm{Zn}$, in $\mathrm{CSF}$ were highlighted and summarized in that review [18].

In the present paper, the metal ion composition of seven CSF samples was measured by using ICP-MS. ICP-MS is widely regarded as one of the best techniques for the characterization of the elemental composition of biological samples. This method was able to provide quantitative results for 33 metals or trace elements, as shown in Table 3 . These data suggest that CSF is a reasonably rich reservoir of trace metals and that ICP-MS can be effectively used to identify and quantify a large number of trace minerals. Furthermore, our experimental results agree reasonably well with the literature values for most of the metal ions except for $\mathrm{Al}, \mathrm{V}, \mathrm{Cr}$, $\mathrm{Ni}$ and As (Table 3). These concentration differences may be due to methodological or equipment differences or they may be due to regional differences with regard to environmental or occupational exposure.

\section{Literature survey of CSF metabolites}

Our literature review allowed us to identify another 57 metabolites that had not been previously archived in the human CSF metabolome database. Furthermore, the literature review also allowed us to update, correct and add more than 500 new concentration ranges or averages.
Additionally, dozens of new disease-metabolite associations were also identified and many older disease-metabolite associations were also updated. A total of 229 disease-metabolite associations are listed in the CSF database. In many cases, multiple concentration values are given for 'normal' conditions, in order to provide the users/readers with a better estimate of the potential concentration variations obtained by different technologies or laboratories. In general, there is good agreement between most laboratories and methods.

\section{The human CSF metabolome - then and now}

The 2008 version of the human CSF metabolome contained 308 fully identified and quantified metabolites [2]. Of these, 70 compounds (or 23\%) were shown to be routinely identifiable using a combination of NMR, GC-MS and ultra performance liquid chromatography UPLCFTMS. Because of the very hydrophilic nature of CSF, it was shown that NMR was the most useful metabolomic platform for characterizing CSF. In 2011, thanks to an extensive literature review and additional experimental analyses, we determined that the human CSF metabolome contains at least 476 fully identified and quantified metabolites. This represents a $54 \%$ increase over the 2008 edition of the CSF metabolome. We were also able to reassess the performance of the previously used 


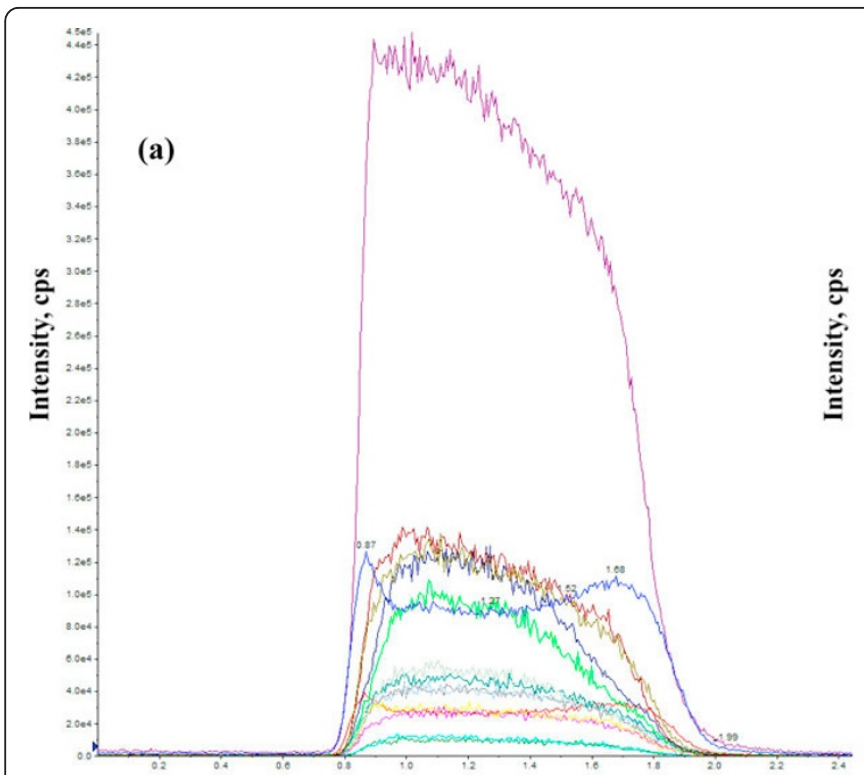

Time, min

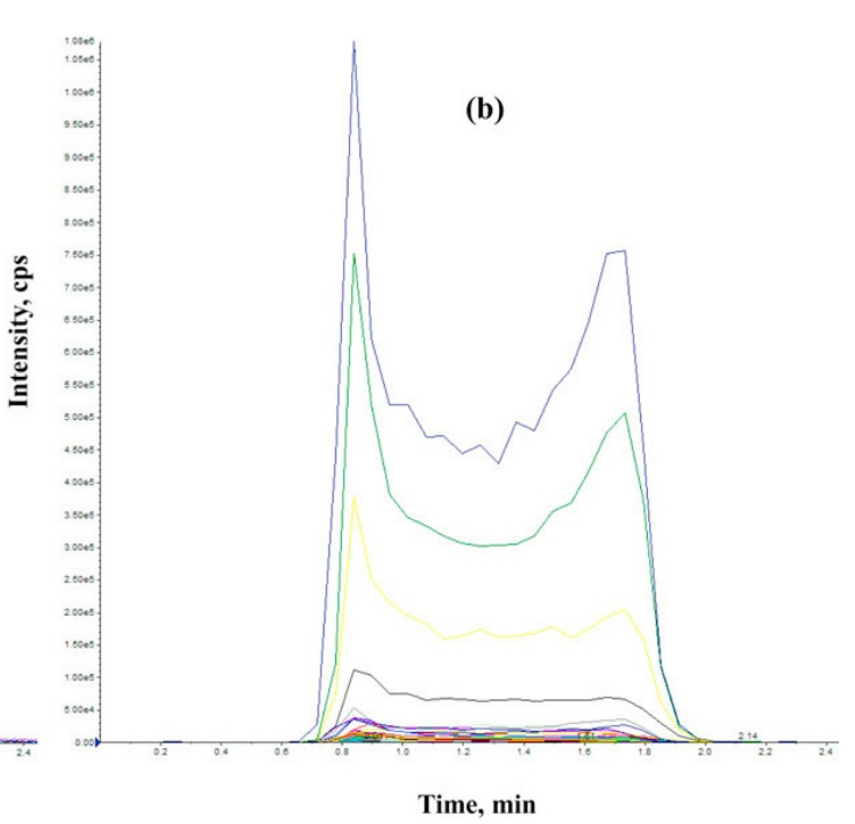

Time, $\min$

Figure 3 Typical direct flow injection (DFI) spectra of human cerebrospinal fluid. (a) negative mode, MRM (15 pairs); (b) positive mode, MRM (175 pairs).

Table 3 Multi-element analysis in cerebral spinal fluid samples using ICP-MS

\begin{tabular}{|c|c|c|c|}
\hline Compound name & Average $(\mu \mathrm{M})$ & Standard deviation $(\mu \mathrm{M})$ & Literature value $(\mu \mathrm{M})$ \\
\hline Lithium (Li) & 10.383 & 4.679 & NA \\
\hline Beryllium (Be) & 0.045 & 0.019 & NA \\
\hline Boron (B) & 3,587 & 1,093 & NA \\
\hline Sodium (Na) & 109,788 & 10,048 & $145,000 \pm 8,000$ \\
\hline Magnesium (Mg) & 954 & 207 & $890 \pm 340$ \\
\hline Aluminum (Al) & 109 & 26 & $12.1 \pm 6.3$ \\
\hline Potassium (K) & 2,163 & 274 & $2,960 \pm 340$ \\
\hline Calcium (Ca) & 1047 & 520 & $1,190 \pm 170$ \\
\hline Titanium (Ti) & 0.648 & 0.168 & NA \\
\hline Vanadium (V) & 0.404 & 0.071 & $0.003 \pm 0.001$ \\
\hline Chromium (Cr) & 0.247 & 0.067 & $0.006 \pm 0.003$ \\
\hline Iron (Fe) & 9.293 & 1.140 & $5.57 \pm 1.68$ \\
\hline Manganese (Mn) & 0.149 & 0.177 & $0.022 \pm 0.031$ \\
\hline Nickel (Ni) & 0.097 & 0.109 & $<0.004$ \\
\hline Copper (Cu) & 2.253 & 3.118 & $1.7 \pm 1.4$ \\
\hline Zinc (Zn) & 0.538 & 0.847 & $0.49 \pm 0.12$ \\
\hline Gallium (Ga) & 0.011 & 0.003 & $<0.001$ \\
\hline Arsenic (As) & 0.106 & 0.010 & $0.003 \pm 0.002$ \\
\hline Selenium (Se) & 0.320 & 0.038 & $0.239 \pm 0.166$ \\
\hline Rubidium (Rb) & 0.499 & 0.097 & $0.620 \pm 0.127$ \\
\hline Strontium (Sr) & 1.120 & 2.481 & $0.534 \pm 0.079$ \\
\hline Yttrium $(Y)$ & 0.004 & 0.001 & NA \\
\hline Zirconium (Zr) & 0.277 & 0.086 & $<0.278$ \\
\hline Molybdenum (Mo) & 0.034 & 0.016 & $0.010 \pm 0.009$ \\
\hline Ruthenium (Ru) & 0.005 & 0.002 & NA \\
\hline Palladium (Pd) & 0.036 & 0.018 & NA \\
\hline Cesium (Cs) & 0.015 & 0.006 & $0.0063 \pm 0.0023$ \\
\hline
\end{tabular}


Table 3 Multi-element analysis in cerebral spinal fluid samples using ICP-MS (Continued)

\begin{tabular}{|c|c|c|c|}
\hline Barium (Ba) & 0.223 & 0.236 & $0.130 \pm 0.050$ \\
\hline Cerium (Ce) & 0.005 & 0.001 & $0.011 \pm 0.005$ \\
\hline Tantalum (Ta) & 0.002 & 0.0009 & $0.0005 \pm 0.0004$ \\
\hline Platinum (Pt) & 0.041 & 0.012 & NA \\
\hline Gold (Au) & 0.060 & 0.063 & $0.196 \pm 0.164$ \\
\hline Lead (Pb) & 0.017 & 0.010 & $0.075 \pm 0.055$ \\
\hline
\end{tabular}

NA, not available.

metabolomic platforms (NMR, GC-MS, UPLC-MS) as well as two more metabolomic platforms (DFI-MS/MS and ICP-MS) with regard to their efficacy in CSF metabolite characterization. Our results indicate that while essentially no improvements could be achieved using the older platforms, the addition of these two newer platforms led to significant improvements. In particular, DFI-MS/MS allowed us to identify and quantify 67 previously unmeasured compounds while ICP-MS allowed another 33 trace metals to be identified and quantified. When combined, the five platforms allowed us to measure 170 of 469 known CSF metabolites (36\%). While the size of the CSF metabolome continues to grow (approximately $13 \%$ a year), the use of improved metabolomic technologies is allowing even greater coverage (growing from $23 \%$ to $36 \%$ ) of the human CSF metabolome. Both trends (that is, the growth in the size of the known metabolome and the growth in metabolome coverage) are encouraging as they indicate that even though our knowledge of the CSF metabolome is rapidly expanding, our ability to characterize it is growing even faster.

\section{Conclusions}

The main objective of this study was to advance the fields of quantitative metabolomics and global metabolic profiling to facilitate future CSF research. The updated CSF metabolome database currently contains 476 detectable metabolites. Our experimental works measured 78 new metabolites that, as per our knowledge, have not been reported to be present in human CSF. This is not a number that will remain unchanged. As technology improves, it is most likely that this number will continue to increase. However, this current set of 476 metabolites appears to provide a reasonably complete listing of the compounds that can be detected and quantified (with today's technology) in the human CSF metabolome.

\footnotetext{
Abbreviations

CSF: cerebral spinal fluid; DFI-MS/MS: direct flow injection-mass spectrometry; FTMS: Fourier transform mass spectrometry; GC-MS: gas chromatography-mass spectrometry; ICP-MS: inductively coupled plasmamass spectrometry; LC-MS: liquid chromatography-mass spectrometry; PC: phosphatidylcholine; MRM: multiple reaction monitoring; NMR: nuclear magnetic resonance; UPLC: ultra performance liquid chromatography.
}

\section{Acknowledgements}

This research was supported by Genome Alberta, Genome Canada, Alberta Advanced Education and Technology (AAET) and the University of Alberta. The authors would like to thank Dr Fiona Bamforth for supplying the CSF samples.

\section{Author details}

'Department of Biological Sciences, University of Alberta, 11455

Saskatchewan Drive, Edmonton, AB Canada T6G 2E8. ²Department of Computing Sciences, University of Alberta, Athabasca Hall, Edmonton, AB Canada T6G 2E8. ${ }^{3}$ National Institute for Nanotechnology, 11421

Saskatchewan Drive, Edmonton, AB, Canada T6G 2 M9.

\section{Authors' contributions}

DSW conceived the project, designed the study and wrote/revised the manuscript; RM designed the experiments, contributed to experiments and data analysis, and wrote/revised the manuscript; ACG constructed the CSF metabolome database and revised the manuscript; KKC, PL, FSY, ED and FA assisted in laboratory experiments and in the CSF metabolome database construction. All authors have read and approved this manuscript for publication.

\section{Competing interests}

The authors declare that they have no competing interests.

Received: 21 January 2012 Revised: 3 April 2012

Accepted: 30 April 2012 Published: 30 April 2012

\section{References}

1. Wishart DS, Tzur D, Knox C, Eisner R, Guo AC, Young N, Cheng D, Jewell K, Arndt D, Sawhney S, Fung C, Nikolai L, Lewis M, Coutouly MA, Forsythe I, Tang P, Shrivastava S, Jeroncic K, Stothard P, Amegbey G, Block D, Hau DD, Wagner J, Miniaci J, Clements M, Gebremedhin M, Guo N, Zhang Y, Duggan GE, Macinnis GD, et al: HMDB: the Human Metabolome Database. Nucleic Acids Res 2007,35 ( D a t a b a s e i s s u e) :

2. Wishart DS, Lewis MJ, Morrissey JA, Flegel MD, Jeroncic $K$, Xiong $Y$, Cheng D, Eisner R, Gautam B, Tzur D, Sawhney S, Bamforth F, Greiner R and Li L: The human cerebrospinal fluid metabolome. J Chrom B 2008, 871:164-173.

3. Hoffmann G, Meier-Augenstein W, Stockler S, Surtees R, Rating D, Nyhan W: Physiology and pathophysiology of organic acids in cerebrospinal fluid. J Inherit Metab Dis 1993, 16:648-669.

4. Toczylowska B, Chalimoniuk M, Wodowska M, Mayzner-Zawadzka E: Changes in concentration of cerebrospinal fluid components in patients with traumatic brain injury. Brain Res 2006, 1104:183-189.

5. Hansson O, Zetterberg H, Buchhave P, Londos E, Blennow K, Minthon L: Association between CSF biomarkers and incipient Alzheimer's disease in patients with mild cognitive impairment: a follow-up study. Lancet Neurol 2006, 5:228-234.

6. Jimenez-Jimenez FJ, Rubio JC, Molina JA, Martin MA, Campos Y, BenitoLeon J, Orti-Pareja M, Gasalla T, Arenas J: Cerebrospinal fluid carnitine levels in patients with Parkinson's disease. J Neurol Sci 1997, 145:183-185.

7. Simone IL, Federico F, Trojano M, Tortiralla C, Liguori M, Giannini P, Piccola E, Natile G, Livrea P: High resolution proton MR spectroscopy of cerebrospinal fluid in MS patients. Comparison with biochemical changes in demyelinating plaques. J Neurol Sci 1996, 144:182-190.

8. HMDB CSF Metabolome Toolbox.. [http://www.csfmetabolome.ca]. 
9. Lutz NW, Cozzone PJ: Metabolic profiling in multiple sclerosis and other disorders by quantitative analysis of cerebrospinal fluid using nuclear magnetic resonance spectroscopy. Curr Pham Biotech 2011, 12:1016-10125.

10. Ng DJY, Pasikanti KK, Chan ECY: Trend analysis of metabonomics and systematic review of metabonomics-derived cancer marker metabolites. Metabolomics 2011, 7:155-178.

11. Blasco H, Corcia P, Moreau C, Veau S, Fournier C, Vourc'h P, Emond P, Gordon P, Pradat PF, Praline J, Devos D, Nadal-Desbarats L, Andres CR: ${ }^{1} \mathrm{H}-$ NMR-Based metabolomic profiling of CSF in early amyotrophic lateral sclerosis. PLOS ONE 2010, 5:e13223.

12. The Automated Mass Spectral Deconvolution and Identification System (AMDIS).. [http://chemdata.nist.gov/mass-spc/amdis/].

13. NIST 08 (2008): Mass spectral library (NIST/EPA/NIH) Gaithersburg, USA: National Institute of Standards and Technology.

14. Wishart DS, Knox C, Guo AC, Eisner R, Young N, Gautam B, Hau DD, Psychogios N, Dong E, Bouatra S, Mandal R, Sinelnikov I, Xia J, Jia L, Cruz JA, Lim E, Sobsey CA, Shrivastava S, Huang P, Liu P, Fang L, Peng J, Fradette R, Cheng D, Tzur D, Clements M, Lewis A, De Souza A, Zuniga A, Dawe M, et al: HMDB: a knowledgebase for the human metabolome. Nucleic Acids Res 2009, 37 (Database issue): D603-610.

15. Bogumil R, Rohring C, Dammeier S, Namendorf C, Uhr M: Targeted Metabolomics Analysis of Cerebrospinal Fluid Using the AbsolutelDQTM Kit. Application Note 1003-1 Innsbruck, Austria: Biocrates Life Sciences;[http:// www.biocrates.com/images/stories/pdf/Folders/Factsheets_etc/biocrates\% 20application\%20note\%201003-1_csf.pdf].

16. Cava-Montesinos P, Cervera ML, Pastor A, de la Guardia M : Room temperature acid sonication ICP-MS multielemental analysisof milk. Anal Chim Acta 2005, 531:111-123.

17. Molina-Holga F, Hider RC, Gaeta A, Williams R, Francis P: Metals ions and neurodegeneration. Bioimetals 2007, 20:639-654.

18. Michalke B, Nischwitz V: Review on metal speciation in cerebrospinal fluid - current methods and results: a review. Anal Chim Acta 2010, 682:23-36.

doi: $10.1186 / g m 337$

Cite this article as: Mandal et al:: Multi-platform characterization of the human cerebrospinal fluid metabolome: a comprehensive and quantitative update. Genome Medicine 2012 4:38.

\section{Submit your next manuscript to BioMed Central and take full advantage of:}

- Convenient online submission

- Thorough peer review

- No space constraints or color figure charges

- Immediate publication on acceptance

- Inclusion in PubMed, CAS, Scopus and Google Scholar

- Research which is freely available for redistribution

Submit your manuscript at www.biomedcentral.com/submit
Biomed Central 